1:256 (cut off <1:64). Ehrlichia and Lyme serology were negative. Significant improvement in symptoms occurred within 3 weeks of starting antibiotic therapy, and a repeat CSD titer was 1:128. After 1 year follow-up and gradual tapering of antiepileptic drugs the patient remains seizure-free. Brain MRI and SPECT are now normal. (Nowakowski GS, Katz A. Epilepsia partialis continua as an atypical presentation of cat scratch disease in a young adult. Neurology December (1 of 2) 2002;59:1815-1816). (Reprints: Dr GS Nowakowski, Mayo Clinic, 200 First St, SW, Rochester, MN 55905).

COMMENT. Encephalopathy and seizures are an unusual presentation of cat scatch disease, and symptoms may be delayed for several weeks after the kitten scratch or bite. Diagnosis is dependent on the history of kitten exposure and positive serology. Spontaneous recovery usually occurs after several days without the need for antibiotics. In the present case, symptoms persisted for several weeks and recovery was prompt only after antibiotics were initiated. The differential diagnosis includes Lyme encephalitis which may occur concurrently. (See Progress in Pediatric Neurology II pp 421-423, for review of 4 previous articles on neurologic complications of cat scratch disease.

\title{
GENETICS OF SIMPLE FEBRILE SEIZURES
}

A clinical and genetic study of three families with simple febrile seizures (FS) and an autosomal dominant (AD) trait with high penetrance is reported from the Hopital Pitie-Salpetriere, Paris, France. Among 29 affected family members, FS ceased before 5 years of age, only one had rare afebrile seizures in addition, and none developed epilepsy. A genome-wide scan in two famiies identified a new locus on chromosome $6 \mathrm{q} 22-\mathrm{q} 24$. This linkage was absent in the third family, supporting genetic heterogeneity of the $\mathrm{AD}$ form of simple FS. (Nabbout $\mathrm{R}$, Prud'homme J-F, Herman A et al. A locus for simple pure febrile seizures maps to chromosome 6q22-q24. Brain December 2002;125:2668-2680). (Respond: Rima Nabbout MD, INSERM U289, Hopital Pitie-Salpetriere, 47 boulevard de l'Hopital, 75013 Paris, France).

COMMENT. This mapping to $6 \mathrm{q} 22-\mathrm{q} 24$ is the first identified locus responsible for simple febrile seizures. Identification of the gene is ongoing. This phenotype differs from the known loci reported for FS and GEFS+. All modes of inheritance have been described, autosomal dominant, autosomal recessive and polygenic.

A nonsense mutation of the MASS1 gene is reported in a family with febrile and afebrile seizures, from University of Tsukuba, Ibaraki, Japan (Nakayama J et al. Ann Neurol Nov 2002;52:654-657).

\section{ABSENCE EPILEPSY AND PAROXYSMAL DYSKINESIA}

Six patients aged 6 to 27 years (mean, 14 years) with childhood absence epilepsy and paroxysmal dyskinesia (PD), identified at five European centers participating in a study group, are reported from Great Ormond Street Hospital, London, UK. The onset of absence seizures was early, at a mean age of 16 months (range, 3 months to 3 years 6 months), and seizures remitted between age 8 and 13 years. The types of associated PD included paroxysmal kinesigenic dyskinesia ( 1 patient), paroxysmal exercise-induced dystonia ( 3 patients), and paroxysmal tonic upgaze (two siblings). Apart from the siblings with tonic upgaze which had an earlier onset, PD developed after the onset of absence seizures, and continued after seizures had remitted. PD improved with increasing age and was not severely disabling. Seizures and PD were idiopathic and were thought to be genetic. Seizures were accompanied by a characteristic $3 \mathrm{~Hz}$ spike-and-wave EEG and they responded to ethosuximide. (Guerrini R, Sanchez-Carpintero R, Deonna T 
et al. Early-onset absence epilepsy and paroxysmal dyskinesia. Epilepsia Oct 2002;43:1224-1229). (Reprints: Prof R Guerrini, Institute of Child Health and Great Ormond Street Hospital, London WC1N 2AP, UK).

COMMENT. Absence epilepsy and paroxysmal dyskinesias may coexist. The age at onset of absence seizures and $3 \mathrm{~Hz}$ spike-and-wave in this comorbid syndrome is unusually early, often in infancy.

\section{RISK OF OVULATORY FAILURE WITH EPILEPSY}

The association of ovulatory dysfunction with epilepsy and antiepileptic drugs (AEDs) was evaluated in women aged 18 to 40 years not receiving hormones recruited from the Stanford and Columbia Universities Comprehensive Epilepsy Centers and from other sources. Patients were followed for three menstrual cycles, a transvaginal ovarian ultrasound was obtained, and multiple endocrine and metabolic factors, including luteinizing hormone were sampled over 8 hours on days 2 to 5 of one cycle. Anovulatory cycles occurred in $10.5 \%$ of cycles in control patients without epilepsy (23), $14.3 \%$ of cycies with localization-related epilepsy (59 patients), and $27.1 \%$ of cycles with idiopathic (primary) generalized epilepsy (35 patients). At least one anovulatory cycle occurred in $38.1 \%$ of women with epilepsy who were taking valproate currently or within 3 years, and in $10.7 \%$ of non-valproate medicated patients. Risk factors for ovulatory dysfunction include idiopathic generalized epilepsy, exposure to valproate AED, high free testosterone, and reduced luteinizing hormone pulses. Patients with polycysticappearing ovaries ( $41 \%$ of those with idiopathic generalized epilepsy cf $16 \%$ women without epilepsy) are not at increased risk and may ovulate normally. (Morrell MJ, Giudice L, Flynn KL et al. Predictors of ovulatory failure in women with epilepsy. Ann Neurol December 2002;52:704-711). (Respond: Dr Martha J Morrell, The Neurological Institute, Department of Neurology, 710 West 168th Street, New York, NY 10032).

COMMENT. Women with idiopathic generalized epilepsy are at increased risk for ovarian dysfunction, anovulatory cycles, and polycystic-appearing ovaries. Those treated with valproate, a cytochrome P450 enzyme inhibiting AED, are at highest risk, and this AED has an additive adverse effect. Cytochrome P450 inducing AEDs (carbamazepine, phenytoin, phenobarbital), and AEDs with no effect on cP450 (lamotrigine, gabapentin) have no adverse additive effect on ovarian function. Ovarian function should be monitored in women with epilepsy. The anovulatory cycles may be the only sign of reproductive dysfunction.

\section{PERIODIC SYNDROMES}

\section{DIAGNOSIS OF CYCLIC VOMITING}

In a study at Children's Hospital, Ann Arbor, MI, and Children's Memorial Hospital, Chicago, designed primarily to compare cost-effectiveness of three methods of management of cyclic vomiting cases, one group of patients received an extensive diagnostic evaluation, a second was treated with empiric antimigraine drugs for 2 months, and a third an upper GI series with small-bowel follow-through (UGI-SBFT) plus empiric therapy. Cyclic vomiting is defined as $>3$ episodes of vomiting within a 3 month period, peak intensity averaging 6 emeses per hour, and intervals of normal health averaging 2 to 4 weeks. The most costeffective approach was the UGI-SBFT followed by antimigraine therapy. A CT scan before antimigraine therapy to rule out a brain tumor would be cost-saving, even 